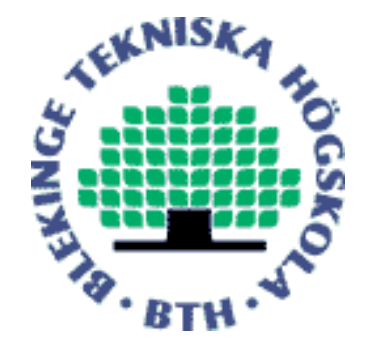

Copyright (C) 2008 IEEE.

Citation for the published paper:

Pareto Optimal Weighting of Structural Impairments for Wireless Imaging Quality Assessment

Ulrich Engelke, Hans-Jürgen Zepernick

15TH IEEE INTERNATIONAL CONFERENCE ON IMAGE PROCESSING, VOLS 1-5

2008 San Diego

This material is posted here with permission of the IEEE. Such permission of the IEEE does not in any way imply IEEE endorsement of any of BTH's products or services Internal or personal use of this material is permitted. However, permission to reprint/republish this material for advertising or promotional purposes or for creating new collective works for resale or redistribution must be obtained from the IEEE by sending a blank email message to pubs-permissions@iee.org.

By choosing to view this document, you agree to all provisions of the copyright laws protecting it. 


\title{
PARETO OPTIMAL WEIGHTING OF STRUCTURAL IMPAIRMENTS FOR WIRELESS IMAGING QUALITY ASSESSMENT
}

\author{
Ulrich Engelke and Hans-Jürgen Zepernick
}

\author{
Blekinge Institute of Technology \\ PO Box 520, SE-372 25 Ronneby, Sweden \\ E-mail: \{ulrich.engelke, hans-jurgen.zepernick\}@bth.se
}

\begin{abstract}
In this paper, we consider multiobjective optimization for obtaining the feature weights associated with structural image impairments and its application to wireless imaging quality assessment. The proposed framework supports optimization with respect to finding a trade-off between metric prediction accuracy and generalization to unknown images. Evaluation of optimal trade-off solutions for two representative scenarios reveal the benefits of the proposed approach. In particular, quality prediction accuracy of an objective image metric can be strongly increased and negligible features are identified that can be discarded to save computational complexity.
\end{abstract}

Index Terms - Multiobjective optimization, objective image quality metrics, wireless image communication.

\section{INTRODUCTION}

Lossy source coding and transmission over wireless channels induce a wide range of artifacts in image and video communication. It is desirable to measure the artifacts accurately and determine their impact on visual perception to define objective metrics that can automatically assess wireless imaging quality. The peak signal-to-noise ratio (PSNR) is not applicable here as it depends on the reference image to be available at the receiver. It also has been shown that the human visual system (HVS) is well adapted to extraction of structural information [1]. A favorable metric would hence be able to judge the quality of a received image using structural rather than pixel based measures, without the need for the reference image. A metric that belongs to this class of reduced-reference objective image quality metrics has been proposed in our earlier work [2,3]. It is based on extraction and summation of a set of low-bandwidth structural image features. Each feature can be weighted according to its impact on the overall metric.

In contrast to this earlier work, in which the weights were obtained separately for each feature, we consider in this paper a multiobjective optimization approach [4] that facilitates finding the optimal weights of all features simultaneously and with respect to two objectives. In this way, interdependencies between the impact of artifacts on perceptual image quality can be accounted for and insights into the perceptual relevance of common artifacts observed in wireless imaging are gained. In addition, multiobjective optimization can be performed for a wide range of system constraints and scenarios of interest. In particular, optimal feature weights are obtained as a trade-off between metric prediction accuracy and generalization to unknown images. An exponential mapping function further increases the metric prediction performance by accounting for the non-linearities in the HVS.

The paper is organized as follows. Section 2 reviews the objective image quality metric and subjective quality experiments. Section 3 and 4, respectively, present and evaluate the multiobjective optimization. Section 5 concludes the paper.

\section{PERCEPTUAL IMAGE QUALITY ASSESSMENT}

\subsection{Objective image quality metric}

We briefly summarize our previously proposed objective metric $[2,3]$ which is based on extraction of five structural features $\tilde{f}_{i}$, in particular, blocking $\tilde{f}_{1}$ [5], blur $\tilde{f}_{2}$ [6], edge-based image activity $\tilde{f}_{3}$ and gradient-based image activity $\tilde{f}_{4}$ [7], and intensity masking $\tilde{f}_{5}$. To obtain a defined feature space, the features were scaled using an extreme value normalization

$$
f_{i, k}=\frac{\tilde{f}_{i, k}-\min _{k=1, \cdots, K}\left\{\tilde{f}_{i, k}\right\}}{\delta_{i}}, \quad i=1, \cdots, I
$$

where the denominator is given as

$$
\delta_{i}=\max _{k=1, \cdots, K}\left\{\tilde{f}_{i, k}\right\}-\min _{k=1, \cdots, K}\left\{\tilde{f}_{i, k}\right\}
$$

Here, $K$ is the number of images in the set and $I$ is the number of features. Resulting from the normalization, we have $\forall i, k$ : $0 \leq f_{i, k} \leq 1$. The feature measures are then accumulated to form the Normalized Hybrid Image Quality Metric (NHIQM)

$$
N H I Q M=\sum_{i=1}^{I} w_{i} \cdot f_{i}
$$

The weights $w_{i}$ represent the impact of an artifact on the overall quality metric and will be optimized according to the perceptual relevance of the corresponding feature. To measure 
structural degradation between a distorted image (d) and its corresponding reference (r), we define an absolute difference

$$
\Delta_{N H I Q M}=\left|N H I Q M_{d}-N H I Q M_{r}\right|
$$

Finally, an exponential function can be used to map $\Delta_{N H I Q M}$ to predicted mean opinion score (MOS) as follows

$$
M O S_{N H I Q M}=a e^{b \Delta_{N H I Q M}}
$$

The exponential character of the prediction function has been found to account well for non-linearities in the HVS.

\subsection{Subjective image quality experiments}

The multiobjective metric optimization is supported by using MOS obtained in subjective quality experiments from two independent laboratories. One experiment was conducted at Blekinge Institute of Technology (BIT) in Ronneby, Sweden, and the other at the Western Australian Telecommunications Research Institute (WATRI) in Perth, Australia [2]. Each experiment involved 30 non-expert viewers. The experiments were designed according to ITU-R Rec. BT.500-11 [8]. A set $\mathcal{I}_{R}$ of 7 reference monochrome images of dimensions $512 \times$ 512 pixels was chosen to account for different textures and complexities. The images were encoded into Joint Photographic Experts Group (JPEG) format. A simulation model of a wireless system was used to generate two sets $\mathcal{I}_{B}$ and $\mathcal{I}_{W}$ of 40 distorted images each, for BIT and WATRI experiments, respectively. In particular, blocking, blur, ringing, and intensity masking artifacts were observed in different degrees of severity. The viewers were shown the distorted images along with their reference images. The experiments at BIT and WATRI resulted in two respective sets of MOS, $\mathcal{M}_{B}$ and $\mathcal{M}_{W}$.

\section{MULTIOBJECTIVE METRIC OPTIMIZATION}

\subsection{Multiobjective optimization}

Optimization in general is concerned with minimization of an objective subject to a set of decision variables. However, the performance of a system cannot always be quantified by a single number. Therefore, multiobjective optimization (MOO) is concerned with the optimization of multiple, often conflicting objectives [4]. Two objectives are said to be conflicting when a decrease in one objective leads to an increase in the other. A MOO problem can be transformed into a single-objective optimization, for instance by defining an objective as a weighted sum of multiple objectives. However, it is recommended to preserve the full dimension of the MOO and instead perform a two stage process [9]. In a first step, the design space is reduced to a set of optimal trade-offs between the objectives by determining the Pareto optimal (noninferior) solutions, which have the characteristic that one objective can only be optimized on cost of another. However, although a Pareto optimal solution should always be a better compromise than the

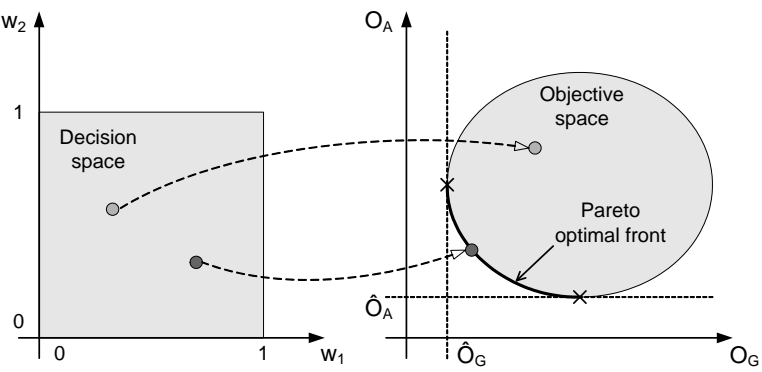

Fig. 1. Multiobjective optimization.

solutions it dominates, not all Pareto optimal solutions may be acceptable solutions. Therefore, in a second step the best trade-off solution is chosen from the set of Pareto optimal solutions under consideration of system design aspects [4].

\subsection{Determination of Pareto optimal weights}

Considering the above, we conduct a two stage MOO with the first step to find the Pareto optimal solutions. We have a decision vector $\mathbf{w}=\left[w_{1}, \ldots, w_{5}\right] \in \mathbb{W} \subset \mathbb{R}^{5}$ containing the feature weights. The range of the weights in the decision space $\mathbb{W}$ is constrained to $w_{i} \in[0,1]$. We define two objectives as: a) maximizing image quality prediction accuracy $O_{A}$ and b) maximizing generalization performance $O_{G}$. Therefore, we randomly created two sets of images, a training (T) set $\mathcal{I}_{T}$ and a validation $(\mathrm{V})$ set $\mathcal{I}_{V}$. The training set contains 60 images, 30 from each $\mathcal{I}_{B}$ and $\mathcal{I}_{W}$. The validation set contains the residual 20 images. Similarly we have created corresponding MOS training set $\mathcal{M}_{T}$ and validation set $\mathcal{M}_{V}$.

Objective $O_{A}$ defines the metrics ability to predict MOS with minimal error and is measured as the Pearson linear correlation between metric $\Delta$ and MOS $\mathcal{M}$ on the training set

$$
\rho_{P}=\frac{\sum_{k}\left(\Delta_{k}-\bar{\Delta}\right)\left(\mathcal{M}_{k}-\overline{\mathcal{M}}\right)}{\sqrt{\sum_{k}\left(\Delta_{k}-\bar{\Delta}\right)^{2}} \sqrt{\sum_{k}\left(\mathcal{M}_{k}-\overline{\mathcal{M}}\right)^{2}}}
$$

Optimizing the weights based only on objective $O_{A}$ would likely overtrain the metric, meaning, it would work very well on the training set but not on a set of unknown images. Therefore, objective $O_{G}$ defines the metrics ability to perform quality prediction on a set of unknown images. We compute it as the absolute difference of $\rho_{P}$ on training and validation set

$$
\Delta \rho_{P}=\left|\rho_{P, T}-\rho_{P, V}\right|
$$

We thus define the objective vector as

$$
\mathbf{O}(w)=\left(\begin{array}{c}
O_{A}(w) \\
O_{G}(w)
\end{array}\right)=\left(\begin{array}{c}
-\left|\rho_{P, T}\right| \\
\Delta \rho_{P}
\end{array}\right)
$$

The decision vector $\mathbf{w}$ is evaluated by assigning it an objective vector $\mathbf{O}$ in the objective space $\mathbb{O}: \mathbb{W} \rightarrow \mathbb{O} \subset \mathbb{R}^{2}$. This is illustrated in Fig. 1 for both two-dimensional decision space and objective space. A decision vector $\mathbf{w}$ is optimal in the 
Pareto sense if it is assigned a noninferior solution on the Pareto optimal front, which is enclosed by $\hat{O}_{A}$ and $\hat{O}_{G}$ as independent optimal solutions for each of the objectives.

We determine the noninferior solutions using goal attainment [10]. Here, goals $\mathbf{O}^{*}=\left(O_{A}^{*} O_{G}^{*}\right)^{T}$ are specified, which can be interpreted as the desired level of the corresponding objective. This requires sufficient intuitive understanding of the problem to know what values one would like to attain for each of the objectives. We then define the MOO problem as

$$
P_{G}:\left\{\begin{array}{cl}
\min & z \\
\text { s.t. } & \mathbf{O}(w)-\boldsymbol{\lambda} \cdot z \leq \mathbf{O}^{*}
\end{array}\right.
$$

with $\boldsymbol{\lambda}=\left(\lambda_{A} \lambda_{G}\right)^{T}$. The magnitude of $\lambda_{j}$ determines how close objective $O_{j}(w)$ is to the goal $O_{j}^{*}$ and is set to $\lambda_{j}=$ $\left|O_{j}^{*}\right|$. The quantity $\boldsymbol{\lambda} \cdot z$ corresponds to the degree of underor overattainment of the goals $\mathbf{O}^{*}$. With regards to results from our earlier metric design [3], we define the goals as $O_{A}^{*}=-0.87$ and $O_{G}^{*} \in[0.001,0.1]$. Higher values than 0.1 were not considered since generalization would be too weak. The noninferior solutions in terms of the Pareto optimal front are shown in Fig. 2. One can see that prediction accuracy on the training set improves as generalization is relaxed. The corresponding optimal weights are shown in Fig. 3. The weights of three features are clearly dominating, namely, blocking, edge-based image activity, and intensity masking. The weights for gradient-based image activity are small over the whole range and the blur weights are all zero. This might be due to the nature of the JPEG codec that mainly produces blocking rather than blur artifacts. Also, blocking usually is perceived more annoying than blur.

\subsection{Exponential mapping to predicted MOS}

For all sets of Pareto optimal weights as in Fig. 3, we conducted non-linear curve fittings of the respective $\Delta_{N H I Q M}$ on the training set and $\mathcal{M}_{T}$ to obtain parameters $a$ and $b$ for the exponential mapping function in (5). Subsequently, all $\Delta_{N H I Q M}$ of both training and validation set were mapped to predicted MOS MOS $S_{N H Q M}$. The Pearson linear correlation $\rho_{P}^{\prime}$ between $M O S_{N H I Q M}$ and MOS for both training and validation set is shown in Fig. 4 over the range of $\Delta \rho_{P}$.

\section{EVALUATION OF TRADE-OFF SOLUTIONS}

In Chapter 3 we determined the Pareto optimal solutions as suggestions for optimal trade-offs between the objectives. We now discuss two representative solutions $S 1$ and $S 2$. The corresponding metrics $\Delta_{N H I Q M, S 1}$ and $\Delta_{N H I Q M, S 2}$ are compared to $\Delta_{N H I Q M, U}$ which is computed using uniform $(U)$ feature weights $\forall i: w_{i}=1$. For further comparison, a structural similarity index (SSIM) [1], a reduced-reference image quality assessment (RRIQA) [11], and the well known PSNR are computed. We derived predicted MOS for all metrics following the procedure in Section 3.3. The metrics are eval-

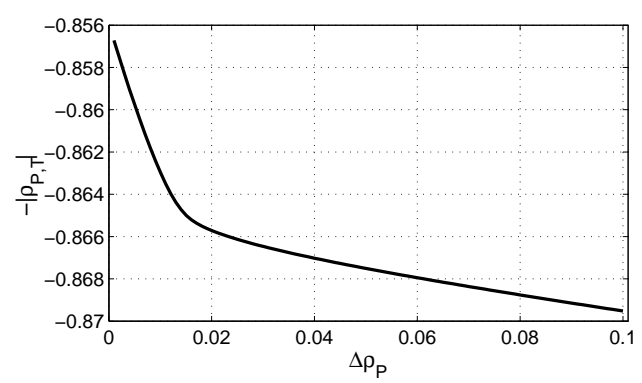

Fig. 2. Pareto optimal front.

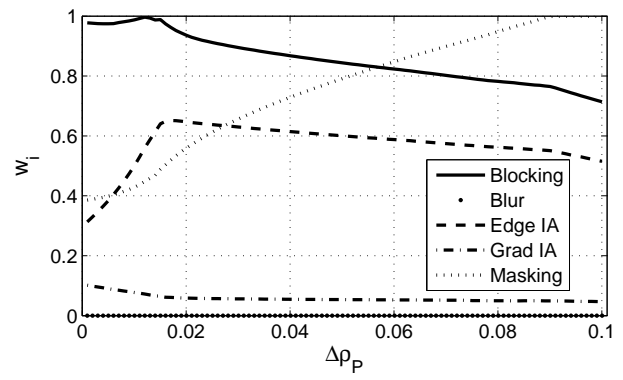

Fig. 3. Pareto optimal weights.

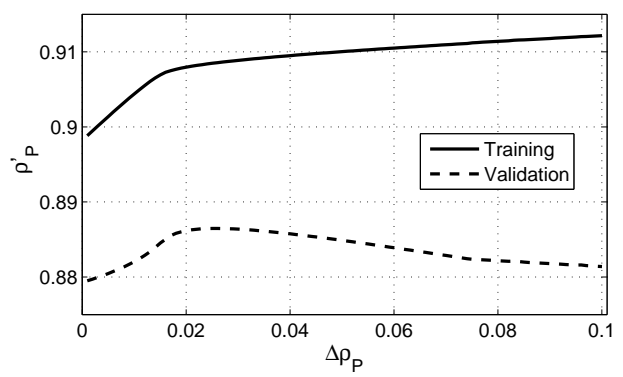

Fig. 4. Pearson correlation $\rho_{P}^{\prime}$ of $\operatorname{MOS}$ and $M O S_{N H I Q M}$.

uated with respect to prediction accuracies $\rho_{P}$ and $\rho_{P}^{\prime}$, respectively, on the actual metric and the corresponding predicted MOS. Additionally, we provide the Spearman rank order correlation coefficient $\rho_{S}$ which measures monotonicity of a metric based on its rank and the rank of MOS. All results are provided in Table 1 for both training and validation sets.

\subsection{Solution $S 1$ : Optimal trade-off for $\Delta_{N H I Q M}$}

Solution $S 1$ was selected with respect to an optimal tradeoff for $\Delta_{N H I Q M}$, disregarding the exponential mapping to $M O S_{N H I Q M}$. When consulting the Pareto optimal front in Fig. 2, it can be seen that the gain in prediction accuracy $\left|\rho_{P, T}\right|$ is small in comparison to the loss in generalization $\Delta \rho_{P}$, as one proceeds along the abscissa. Accordingly, the optimal trade-off for $\Delta_{N H I Q M}$ optimization has been chosen at $\Delta \rho_{P}=0.001$ representing the best generalization ability. The corresponding weights are then obtained as

$w_{1}^{(1)}=0.98, w_{2}^{(1)}=0, w_{3}^{(1)}=0.31, w_{4}^{(1)}=0.1, w_{5}^{(1)}=0.39$. 


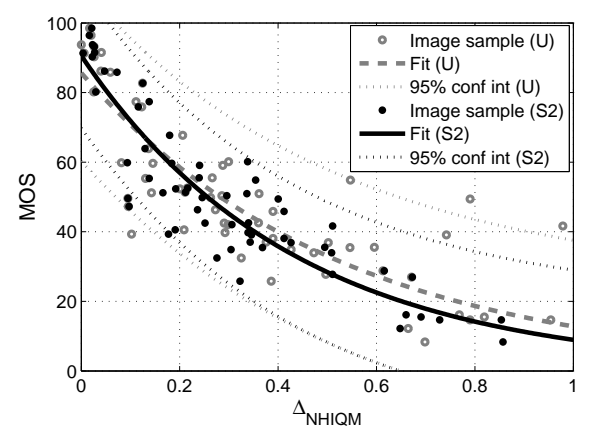

Fig. 5. Exponential curve fitting of MOS with $\Delta_{N H I Q M}$.

This set of weights $w_{i}^{(1)}$ also provides direct insight into the perceptual relevance of wireless imaging artifacts, since we only considered the linear relationship of $\Delta_{N H I Q M}$ between the weights, rather than the non-linearity of $M O S_{N H I Q M}$. In Table 1 it is shown that $\Delta_{N H I Q M, S 1}$ strongly outperforms $\Delta_{N H I Q M, U}$ on both prediction accuracies $\rho_{P, T}$ and $\rho_{P, V}$.

\subsection{Solution $S 2$ : Optimal trade-off for $M O S_{N H I Q M}$}

Given the previous results, solution $S 2$ was chosen with respect to an optimal trade-off for $M O S_{N H I Q M}$. In Fig. 4 one can see that $\rho_{P}^{\prime}$ for the training set continuously increases with $\Delta \rho_{P}$. The validation set, however, has a maximum of $\rho_{P}^{\prime}$ at $\Delta \rho_{P}=0.026$ and has thus been chosen as the best trade-off for $M O S_{N H I Q M}$. The corresponding weights are given by

$w_{1}^{(2)}=0.91, w_{2}^{(2)}=0, w_{3}^{(2)}=0.64, w_{4}^{(2)}=0.06, w_{5}^{(2)}=0.62$.

Table 1 reveals that $M O S_{N H I Q M, S 2}$ has improved prediction accuracies $\rho_{P, T}^{\prime}$ and $\rho_{P, V}^{\prime}$ compared to $M O S_{N H I Q M, S 1}$. The improvement to the uniform case $M O S_{N H I Q M, U}$, however, is not as significant as one might have expected. This can be comprehended when analyzing the exponential curve fitting in Fig. 5. Even though the fit of $\Delta_{N H I Q M, S 2}$ has a root mean square error of only 9.9 as compared to 12.2 for $\Delta_{N H I Q M, U}$, it can be observed that both $\Delta_{N H I Q M, S 2}$ and $\Delta_{N H I Q M, U}$ produce similar mapping functions. However, it should be noted that $M O S_{N H I Q M, S 2}$ delivers comparable prediction accuracy to $M O S_{N H I Q M, U}$ without the blur metric and thus saves valuable computational complexity.

\section{CONCLUSIONS}

In this paper, multiobjective optimization has been utilized to determine feature weights related to common wireless imaging impairments. The optimized weights enabled us to improve the prediction accuracy of our objective image quality metric and to save computational complexity by discarding features associated with negligible weights. Additionally, insight was revealed into the perceptual relevance of common artifacts in wireless imaging. The paper may also serve as a guide to multiobjective optimization of image quality metrics.
Table 1. Prediction accuracy $\rho_{P}$ and monotonicity $\rho_{S}$.

\begin{tabular}{|c|c||cc|cc||cc|}
\hline \multicolumn{2}{|c|}{} & \multicolumn{5}{c||}{ Pearson } & \multicolumn{2}{c|}{ Spearman } \\
\cline { 3 - 8 } \multicolumn{2}{|c||}{} & $\rho_{P, T}$ & $\rho_{P, V}$ & $\rho_{P, T}^{\prime}$ & $\rho_{P, V}^{\prime}$ & $\rho_{S, T}$ & $\rho_{S, V}$ \\
\hline \hline \multirow{3}{*}{ NHIQM } & $U$ & 0.795 & 0.781 & 0.859 & 0.909 & 0.848 & 0.899 \\
\cline { 2 - 8 } & $S 1$ & 0.857 & 0.856 & 0.899 & 0.879 & 0.875 & 0.902 \\
\cline { 2 - 8 } & $S 2$ & 0.866 & 0.840 & 0.909 & 0.887 & 0.876 & 0.892 \\
\hline \hline \multicolumn{2}{|c|}{ SSIM } & 0.582 & 0.434 & 0.632 & 0.511 & 0.558 & 0.347 \\
\hline \multicolumn{2}{|c|}{ RRIQA } & 0.821 & 0.772 & 0.829 & 0.749 & 0.786 & 0.758 \\
\hline \multicolumn{2}{|c|}{ PSNR } & 0.742 & 0.712 & 0.738 & 0.710 & 0.638 & 0.615 \\
\hline
\end{tabular}

\section{REFERENCES}

[1] Z. Wang, A. C. Bovik, H. R. Sheikh, and E. P. Simoncelli, "Image quality assessment: From error visibility to structural similarity," IEEE Trans. on Image Processing, pp. 600-612, April 2004.

[2] T. M. Kusuma, H.-J. Zepernick, and M. Caldera, "On the development of a reduced-reference perceptual image quality metric," in Proc. of ICMCS, Aug. 2005, pp. $178-184$.

[3] U. Engelke and H.-J. Zepernick, "Quality evaluation in wireless imaging using feature-based objective metrics," in Proc. of IEEE ISWPC, Feb. 2007, pp. 367-372.

[4] C. M. Fonseca and P. J. Fleming, "Multiobjective optimization," in Evolutionary Computation 2: Advanced Algorithms and Operations, T. Back, Ed., chapter 5, pp. 25-37. Taylor \& Francis, 2000.

[5] Z. Wang, H. R. Sheikh, and A. C. Bovik, "No-reference perceptual quality assessment of JPEG compressed images," in Proc. of IEEE ICIP, Sept. 2002, pp. 477-480.

[6] P. Marziliano et al, "A no-reference perceptual blur metric," in Proc. of IEEE ICIP, Sept. 2002, pp. 57-60.

[7] S. Saha and R. Vemuri, "An analysis on the effect of image features on lossy coding performance," IEEE Signal Processing Letters, pp. 104-107, May 2000.

[8] ITU, "Methodology for the subjective assessment of the quality of television pictures," Rec. BT.500-11, 2002.

[9] L. A. Zadeh, "Optimality and non-scalar-valued performance criteria," IEEE Trans. on Automatic Control, vol. 8, no. 1, pp. 59-60, Jan. 1963.

[10] F. W. Gembicki and Y. Y. Haimes, "Approach to performance and sensitivity multiobjective optimization: The goal attainment method," IEEE Trans. on Automatic Control, vol. 20, no. 6, pp. 769-771, Dec. 1975.

[11] Z. Wang and E. P. Simoncelli, "Reduced-reference image quality assessment using a wavelet-domain natural image statistic model," in Proc. of SPIE HV \& EI, Mar. 2005, vol. 5666, pp. 149-159. 